\title{
Expression of truncated Kir6.2 promotes insertion of functionally inverted ATP-sensitive $\mathrm{K}^{+}$channels
}

\author{
Benjamin A. Heitz ${ }^{1,5}$, Robert Bränström ${ }^{3,4,5 凶}$, Wei Yang ${ }^{1}$, Yiding Huang ${ }^{1}$, Tilo Moede ${ }^{3}$, \\ Ingo B. Leibiger ${ }^{3}$, Barbara Leibiger ${ }^{3}$, Liu Qi Chen ${ }^{1}$, Jia Yu ${ }^{3}$, Shao-Nian Yang ${ }^{3}$, Olof Larsson ${ }^{3}$, \\ S. Scott Saavedra ${ }^{1,2}$, Per-Olof Berggren ${ }^{3} \&$ Craig A. Aspinwall ${ }^{1,2}$
}

ATP-sensitive $\mathrm{K}^{+}\left(\mathrm{K}_{\mathrm{ATP}}\right)$ channels couple cellular metabolism to electrical activity in many cell types. Wild-type $K_{\text {ATP }}$ channels are comprised of four pore forming (Kir6.x) and four regulatory (sulfonylurea receptor, SURX) subunits that each contain RKR endoplasmic reticulum retention sequences that serve to properly translocate the channel to the plasma membrane. Truncated Kir6.x variants lacking RKR sequences facilitate plasma membrane expression of functional Kir6.x in the absence of SURx; however, the effects of channel truncation on plasma membrane orientation have not been explored. To investigate the role of truncation on plasma membrane orientation of ATP sensitive $\mathrm{K}^{+}$ channels, three truncated variants of Kir6.2 were used (Kir6.2 $\Delta \mathrm{C} 26,6 x H i s-K i r 6.2 \Delta C 26$, and $6 x H i s-$ EGFP-Kir6.2 $\Delta$ C26). Oocyte expression of Kir6.2 $\Delta$ C26 shows the presence of a population of inverted inserted channels in the plasma membrane, which is not present when co-expressed with SUR1. Immunocytochemical staining of intact and permeabilized HEK293 cells revealed that the $\mathrm{N}$-terminus of $6 x$ His-Kir6.2 $2 \mathrm{C} 26$ was accessible on both sides of the plasma membrane at roughly equivalent

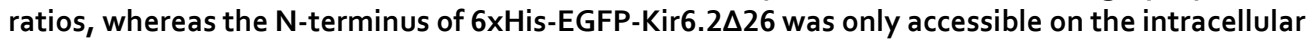
face. In HEK293 cells, whole-cell electrophysiological recordings showed a ca. $50 \%$ reduction in $\mathrm{K}^{+}$ current upon addition of ATP to the extracellular solution for $6 x \mathrm{His}-\mathrm{Kir} 6.2 \Delta \mathrm{C} 26$, though sensitivity to extracellular ATP was not observed in 6xHis-EGFP-Kir6.2 $\Delta$ C26. Importantly, the population of channels that is inverted exhibited similar function to properly inserted channels within the plasma membrane. Taken together, these data suggest that in the absence of SURx, inverted channels can be formed from truncated Kir6.x subunits that are functionally active which may provide a new model for testing pharmacological modulators of Kir6.x, but also indicates the need for added caution when using truncated Kir6.2 mutants.

Adenosine triphosphate (ATP)-sensitive $\mathrm{K}^{+}\left(\mathrm{K}_{\mathrm{ATP}}\right)$ channels are present in a wide range of tissues ${ }^{1-4}$ including pancreatic islet cells ${ }^{4-8}$, heart ${ }^{9-11}$, skeletal muscle ${ }^{12}$, vascular smooth muscle ${ }^{11,13}$, and brain ${ }^{14}$. $K_{\text {ATP }}$ channels serve to couple the metabolic state of the cell to electrical activity ${ }^{6}$. As such, $\mathrm{K}_{\mathrm{ATP}}$ channels play key roles in regulating diverse biological functions such as insulin secretion ${ }^{6,15}$, cardiac action potentials ${ }^{16,17}$, ischemic preconditioning $^{18,19}$, and blood pressure ${ }^{20}$.

$\mathrm{K}_{\text {ATP }}$ channel structure has been investigated in detail in a range of tissues ${ }^{1,3,4,21}$. Cloning and reconstitution of functional $\mathrm{K}_{\mathrm{ATP}}$ channels and channel variants have illuminated key advances in $\mathrm{K}_{\mathrm{ATP}}$ channel structure-function relationships ${ }^{7}$. Structurally, $\mathrm{K}_{\mathrm{ATP}}$ channels are heterooctamers comprised of four pore forming (Kir6.x) subunits from the family of small inwardly rectified $\mathrm{K}^{+}$channels and four regulatory subunits (SURx) from the sulfonylurea receptor family $13,4,21,22$. While most physiological and pharmaceutical ligand sensitivity is imparted by the SURx subunits ${ }^{1,3,4,23}$, ATP, phosphoinositides and long-chain coenzyme A (LC-CoA) esters act via the Kir6.x subunit ${ }^{3,24-29}$. Among the most studied $\mathrm{K}_{\mathrm{ATP}}$ channels are those expressed in the insulin secreting pancreatic $\beta$-cell, where the $K_{A T P}$ channel is comprised of Kir6.2 and SUR1 ${ }^{1,29-31}$. A range of diseases are associated with

\footnotetext{
${ }^{1}$ Department of Chemistry and Biochemistry, University of Arizona, Tucson, AZ 85721, USA. ${ }^{2} \mathrm{BIO} 5$ Institute and Department of Biomedical Engineering, University of Arizona, Tucson, AZ 85721, USA. ${ }^{3}$ The Rolf Luft Research Center for Diabetes and Endocrinology, Karolinska Institutet, Karolinska University Hospital, 17176 Stockholm, Sweden. ${ }^{4}$ Endocrine and Sarcoma Surgery Unit, Department of Molecular Medicine and Surgery, Karolinska Institutet, Karolinska University Hospital, 17176 Stockholm, Sweden. ${ }^{5}$ These authors contributed equally: Benjamin A. Heitz and Robert Bränström. ${ }^{\circledR}$ email: robert.branstrom@ki.se
} 


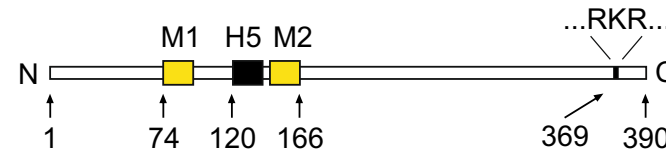

$\mathrm{N}$

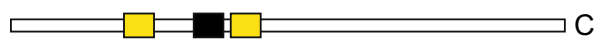

$6 x \mathrm{His}$

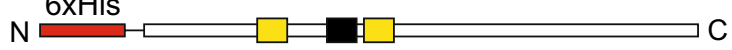

iii

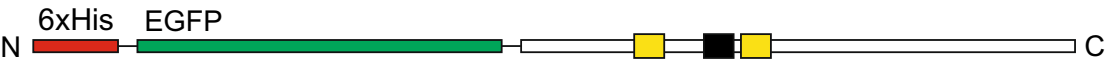

$i v$

Figure 1. Schematic diagram of Kir6.2 variants. Illustration shows the Kir6.2 constructs used with the N-and C-termini deleted and/or fused with 6xHis and EGFP. Full length wild-type Kir6.2 contains 390 amino acids with two transmembrane segments (M1 and M2) and a pore-forming $\mathrm{H} 5$ segment $(i)$. C-terminal truncated Kir6.2, Kir6.2 $\Delta$ C26 (ii), N-terminal 6xHis tag fused with Kir6.2 $\Delta$ C26, 6xHis-Kir6.2 $\Delta$ C26 (iii), and 6xHis and EGFP chimera N-terminal fused with Kir6.2 $\Delta$ C26, 6xHis-EGFP-Kir6.2 $\Delta$ C26 (iv).

either mutation and/or improper trafficking of SURx or Kir6.2 subunits, including type 2 diabetes mellitus ${ }^{8,32,33}$, and persistent hyperinsulinemic hypoglycemia of infancy $(\mathrm{PHHI})^{3,20,34-36}$.

Functional expression of wild-type Kir6.2 in the plasma membrane of model organisms requires co-expression with $\mathrm{SURx}^{7,29}$. Detailed investigation of this phenomenon revealed the presence of an endoplasmic reticulum (ER) retention signal comprised of a three amino acid (RKR) sequence on both Kir6.2 and SURx ${ }^{37}$. Proper trafficking of the $\mathrm{K}_{\mathrm{ATP}}$ channel requires that all RKR sequences, from both subunits, be shielded, which occurs during assembly of the channel complex within the $\mathrm{ER}^{37}$. Further, only functional $\mathrm{K}_{\mathrm{ATP}}$ channels in the proper stoichiometric ratio in the ER can sufficiently mask the RKR retention signal and facilitate export to the Golgi apparatus and eventually the plasma membrane ${ }^{37}$.

The RKR sequence in Kir6.2 is positioned near the C-terminus (AA369-371, Fig. 1) whereas in SUR1 the RKR sequence is positioned near nuclear binding fold-1 (Walker A motif) ${ }^{36,37}$. Mutation of RKR to AAA in SUR1 increases surface expression of sulfonylurea-sensitive $\mathrm{K}^{+}$currents whereas the RKR to AAA mutation in Kir6.2 increases the expression of sulfonylurea-insensitive $\mathrm{K}^{+}$currents due to enhanced Kir6.2 cell-surface expression ${ }^{37}$. Additionally, truncation of Kir6.2, where the last 26 or 36 amino acids (Kir6.2 $\Delta$ C26 or Kir6.2 $\Delta$ C36) containing the RKR signal were removed, facilitates expression of functional $\mathrm{K}^{+}$channel activity that retains Kir6.2 ligand sensitivity in the absence of SUR $1^{26}$. Therefore, the truncated mutants have been used to investigate ligand binding sites and other $\mathrm{K}_{\mathrm{ATP}}$ channel structure-function relationships ${ }^{26,27,38,39}$.

Proper assembly of the $\mathrm{K}_{\text {ATP }}$ channel complex within the ER and subsequent regulated trafficking ensure normal orientation within the cell membrane $e^{37}$. However, little is known regarding the orientation of channels formed from truncated Kir6.2 mutants. This is particularly important since normal orientation is required to maintain ionic balance within the cell. Importantly, the $\mathrm{K}_{\mathrm{ATP}}$ channel can conduct ions in both directions, yielding both inward and outward currents of different magnitudes ${ }^{38,40-43}$; however, the ligand sensitivity is specific to individual faces of the assembled channel. Many studies utilizing Kir6.2 mutants rely on whole-cell currents from mammalian cells or whole-cell or excised macropatch currents from Xenopus oocytes ${ }^{26}$ and thus may not be truly indicative of the population of normally oriented channels and may contain substantial ligand-insensitive background current resulting from abnormally oriented channels. In this work, we have investigated the effects of truncation of RKR on the plasma membrane orientation of Kir6.2 mutants expressed in Xenopus oocytes and mammalian cells using a combination of electrophysiological and immunocytochemical techniques.

\section{Materials and methods}

HEK293 culture. Human embryonic kidney (HEK293) cells, supplied by The American Type Culture Collection (ATCC), were cultured in minimum essential medium (MEM) supplemented with $10 \%$ fetal bovine serum (FBS) and $1 \%$ streptomycin and penicillin and incubated at $5 \% \mathrm{CO}_{2}, 37^{\circ} \mathrm{C}$. Media were changed every 2-3 days. Cells were split at 80-90\% confluency using Puck's EDTA (140 mM NaCl, $5.5 \mathrm{mM} \mathrm{KCl}, 5.5 \mathrm{mM}$ glucose, $4.2 \mathrm{mM} \mathrm{NaHCO}_{3}, 0.5 \mathrm{mM}$ EDTA, $\mathrm{pH} 7.40$ ), followed by trypsin-EDTA with gentle rocking, and harvested with MEM. Cells were then centrifuged at $200 \mathrm{~g}$ for $2 \mathrm{~min}$, resuspended into fresh MEM and placed into new flask at a 1:10 dilution. All media and additives were obtained from Invitrogen.

Xenopus leavis oocytes. For collection of oocytes, large female Xenopus laevis were anaesthetized with 3-aminobenzoic acid methyl ester ( $1.5 \mathrm{~g} / \mathrm{L}$ of water, Sigma) and handled using a previously established protocol $^{44}$. Briefly, oocytes were removed from one ovary by laparotomy, the incision was sutured, and the animal was allowed to recover. Oocytes, stage V-VI, were defolliculated using collagenase A and injected using an Eppendorf transjector (Eppendorf, Hamburg, Germany) with 0.5-5 ng of mRNA/50 nL of sterile RNasefree water, encoding Kir6.2 $\Delta \mathrm{C} 26$ or Kir6.2 $\Delta \mathrm{C} 26$ together with SUR1. Oocytes were maintained in culture at a temperature of $19^{\circ} \mathrm{C}$, and experiments were performed 2-5 days after mRNA injection. Control oocytes were injected with $50 \mathrm{~nL}$ of sterile water. 
Preparation of expression plasmids for Kir6.2 mutants. For HEK293 cells, Kir6.2 mutants were originated from full-length human Kir6.2 cDNA template (courtesy of Dr. Joseph Bryan). The Kir6.2 $\Delta$ C26 gene fragment was cloned into pcDNA4/HisMax-TOPO-TA vector using the TA cloning technique to construct a plasmid for expressing $6 \mathrm{xHis}$-Kir6.2 $\Delta \mathrm{C} 26$ protein in mammalian cells. The sequences of the forward and reverse PCR primers were 5'-GGA TCC ATG CTG TCC CGC AAG GGC ATC-3' and 5'-GGA TCC TCA GGC TGA GGC GAG GGT CAG-3', respectively. The plasmid for expressing 6xHis-EGFP-Kir6.2 $\Delta$ C26 in HEK293 cells was prepared by inserting the Kir6.2 $\Delta$ C26 gene fragment from Kir6.2 cDNA into pET28a(+)/EGFP plasmid ${ }^{45}$ using forward (5'-ATA-GTC GAC AAA TGC TGT CCC GCA AGG GCA T-3') and reverse primer (5'-ATG CGG CCG CAT CAG GCT GAG GCG AGG GTC AGA G-3'). After the pET28a(+)/EGFP-Kir6.2 $\Delta$ C26 template was prepared, the EGFP- Kir6.2 $\Delta$ C26 sequence was amplified using forward (5'-GGA TCC ATG GTG AGC AAG GGC GAG GAG-3') and reverse (5'-GGA TCC TCA GGC TGA GGC GAG GGT CAG-3'), which was subsequently ligated into pcDNA4/HisMax-TOPO-TA vector to form the recombinant plasmid for expressing 6xHis-EGFP-Kir6.2 $\Delta$ C26 in HEK293 cells. Plasmids were propagated in E. coli strain DH5a cultured in LB broth or on agar plates with $50 \mu \mathrm{g} / \mathrm{mL}$ ampicillin/kanamycin. Plasmids were purified using plasmid DNA isolation kits (Promega) and stored in Tris-EDTA (TE) buffer or Nanopure water at $-20^{\circ} \mathrm{C}$. DNA quantity and purity was assessed using UV absorbance spectrophotometry $\left(\mathrm{A}_{260}\right.$ and $\left.\mathrm{A}_{280}\right)$, agarose gel electrophoresis, DNA sequencing (University of Arizona Genetics Core Facility) and restriction digestion.

For oocytes, the cDNAs of mouse Kir6.2 (GenBank accession number D50581) and hamster SUR1 (GenBank accession number L40623) were subcloned into pBluescript II SK (Stratagene, La Jolla, CA), creating pB.mKir6.2 and pB.SUR1, respectively. Plasmid pB.mKir6.2 $\Delta 365-390$ was generated by introducing a stop codon (R365Stop) into pB.mKir6.2 by site-directed mutagenesis using the QuikChange Mutagenesis kit (Stratagene). Capped mRNA was synthesized by in vitro transcription from linearized plasmids employing the mMESSAGE mMACHINE kit (Ambion, Austin, TX). The purified mRNA was dissolved in $10 \mathrm{mM}$ Tris- $\mathrm{HCl}(\mathrm{pH} 7.40)$ and stored in aliquots at $-80^{\circ} \mathrm{C}$ until use.

Transfection of HEK293 cells and selection. Both transient and long-term expressions were initiated similarly. Cells were detached using trypsin and resuspended in fresh media at the following approximate densities: $1.8 \times 10^{4}, 1.0 \times 10^{5}, 4.2 \times 10^{5}, 2.8 \times 10^{6}, 1.3 \times 10^{6}$, and $3.8 \times 10^{6}$ per well for a 96 well plate, 24 well plate, $35 \mathrm{~mm}$ petri dish, $100 \mathrm{~mm}$ petri dish, $25 \mathrm{~cm}^{2}$ flask, or $75 \mathrm{~cm}^{2}$ flask, respectively. Cell densities were further optimized dependent upon cell type and plasmids. Cells were incubated for $24 \mathrm{~h}$ or until $80-90 \%$ confluency was reached for transient expression and 50-60\% for long-term expression. At this point, transfection was performed using Lipofectamine 2000 (Invitrogen). Lipofectamine:DNA ratios were optimized by monitoring protein expression $48 \mathrm{~h}$ following transfection under varying ratios where an optimized ratio of $2.5 \mu \mathrm{L}$ Lipofectamine: $1 \mu \mathrm{g}$ of DNA was identified. Lipofectamine:DNA was added to cells in serum free Opti-MEM media, incubated for $4 \mathrm{~h}$, followed by replacement with serum-supplemented media. Cells were incubated for 24-48 h to facilitate optimum transient protein expression. Long-term expressions were performed using Zeocin selection. Transfected cells were split after $48 \mathrm{~h}$ into new flasks or dishes, allowed to adhere to the surface for 12-24 h, and media replaced with Zeocin-doped media. Initial rounds of selection were typically performed in 24 well plates with a series of concentrations ranging from 50 to $500 \mu \mathrm{g} / \mathrm{mL}$ Zeocin. Media were changed every 2-3 days and cells were split as necessary. Typically $2-3$ splits were performed prior to stable expression.

RT-PCR. Expression of constructs was further verified via reverse transcriptase-PCR (RT-PCR). RNA was isolated from wild-type and transfected (6xHis-Kir6.2, 6xHis-EGFP-Kir6.2 $\Delta$ C26, and 6xHis-Kir6.2 $\Delta$ C26) cells using SV Total RNA isolation kit (Promega). RT-PCR was performed on isolated RNA using AccessQuick RTPCR kit (Promega). Forward and reverse primers for 6xHis-EGFP-Kir6.2 $\Delta \mathrm{C} 26$ and $6 x$ His-Kir6.2 $\Delta \mathrm{C} 26$ were designed: 5'-GCG GCC GCA TGG GGG GTT CTC ATC ATC A-3' (6xHis-Kir6.2 $\Delta$ C26 Forward), 5'-TCT AGA TCA GGC TGA GGC GAG GGT-3' (6xHis-EGFP-Kir6.2 $\triangle$ C26), 5'-GCG GCC GCA TGG GGG GTT CTC ATC ATA-3' (6xHis-Kir6.2 $\Delta$ C26 Forward), and 5'-TCT AGA TCA GGC TGA GGC GAG GGT-3' (6xHisKir6.2 $\Delta$ C26 Reverse). Non-transfected controls utilized each set of primers. All primers were received from Integrated DNA Technologies and diluted into sterile water to prepare $100 \mu \mathrm{M}$ stock concentrations. Working solutions of $10 \mu \mathrm{M}$ were prepared for use in PCR.

Immunocytochemistry. PentaHis-biotin conjugate (Mouse IgG1) (biotinylated anti-6xHis) was purchased from Qiagen (Catalog \#34440). Streptavidin-fluorescein (Catalog \#S869) and anti-GFP AlexaFluor 594 conjugates (Rabbit IgG) (Catalog \#A21312) were purchased from Invitrogen. All antibodies were used as received. Cells were split onto coverslips $24-48 \mathrm{~h}$ prior to immunocytochemical staining. Immediately prior to staining, cells were washed twice with PBS, then fixed at room temperature using $4 \%$ (v/v) formaldehyde in PBS for $60 \mathrm{~min}$. Cell fixation and all subsequent treatments were followed by $2-5$ rinses with PBS. For permeabilized cells, $0.25 \%$ (v/v) Triton X-100 in PBS was added to the cells for 5 min following fixation. Non-specific adsorption was blocked by treatment with 5\% FBS in PBS for $60 \mathrm{~min}$. Expression and orientation of 6xHis-EGFPKir6.2 $\Delta$ C26 were observed via staining with anti-GFP AlexaFluor $594(2 \mu \mathrm{g} / \mathrm{mL})$ in PBS for $60 \mathrm{~min}$. Detection of 6xHis-Kir6.2 $\Delta \mathrm{C} 26$ required primary and secondary stains as follows: fixed cells were incubated with PentaHisbiotin conjugate in PBS for $60 \mathrm{~min}(0.2 \mu \mathrm{g} / \mathrm{mL})$, followed by $1 \mu \mathrm{g} / \mathrm{mL}$ fluorescein-conjugated streptavidin for $60 \mathrm{~min}$. Images were acquired using a using Nikon Eclipse TE300 inverted epifluorescence microscope with a 540/25 excitation filter and 620/60 emission filter for AlexaFluor 594 and 480/30 excitation filter and 535/40 emission filter for EGFP and fluorescein. Images were collected using a Cascade 650 front illuminated CCD camera or MicroMAX 512BFT back illuminated CCD camera (Roper Scientific, Tucson, AZ). MetaVue software 
version 1.0 (Universal Imaging, Downingtown, PA; https://www.moleculardevices.com/) was used to capture images and Image $\mathrm{J}^{46}$ was used to analyze all images.

Electrophysiological recordings. Electrophysiological recordings were used to evaluate channel function and orientation. For HEK293 cells, recordings were collected using a HEKA EPC-8 using Pulse software version 7.0 (HEKA Elektronik Dr. Schulze GmbH, Germany; https://www.heka.com/about/about_main.html\# smart-ephys). Pipettes were pulled from borosilicate glass and yielded a measured resistance between 4-6 M $\Omega$. For whole-cell recordings, the extracellular solution (bath solution) was composed of (in $\mathrm{mM}$ ): $138 \mathrm{NaCl}, 5.6$ $\mathrm{KCl}, 1.2 \mathrm{MgCl}_{2} \cdot 6 \mathrm{H}_{2} \mathrm{O}, 2.6 \mathrm{CaCl}_{2}$, and $5 \mathrm{HEPES}(\mathrm{pH} 7.40$ ). The intracellular solution (pipet solution) was composed of (in mM): $125 \mathrm{KCl}, 1 \mathrm{MgCl}_{2} \cdot 6 \mathrm{H}_{2} \mathrm{O}, 30 \mathrm{KOH}, 10 \mathrm{EGTA}$, and $5 \mathrm{HEPES}$ (pH 7.15).

For oocytes, inside-out recordings of channel activity were obtained at a holding-potential of $-80 \mathrm{mV}$ and pipette solution containing (in $\mathrm{mM}$ ): $140 \mathrm{KCl}, 1.2 \mathrm{MgCl}_{2} \cdot 6 \mathrm{H}_{2} \mathrm{O}, 2.6 \mathrm{CaCl}_{2}, 5 \mathrm{HEPES}$ at $\mathrm{pH} 7.40$, and an internal (bath) solution consisting of (in $\mathrm{mM}$ ) $140 \mathrm{KCl}, 1 \mathrm{MgCl}_{2} \cdot 6 \mathrm{H}_{2} \mathrm{O}, 10 \mathrm{EGTA}, 5 \mathrm{HEPES}$ at $\mathrm{pH}$ 7.15. For outside-out recordings, the solutions were reversed. Recordings were made using an Axopatch 200 (Axon Instrument, CA). Channel records are displayed according to the convention that upward deflections denote outward currents and vice versa. The experiments were carried out at room temperature of $20-22^{\circ} \mathrm{C}$. All solutions were prepared in deionized water and filtered through a $0.2 \mu \mathrm{m}$ pore-size filter prior to use. ATP was added as $\mathrm{Mg}^{2+}$-salt, and all reagents were of analytical grade and obtained from Sigma-Aldrich.

Statistical analysis. For electrophysiological recordings, each group vs. control were compared using the Student's t-test. All data are reported as mean \pm SD.

\section{Results and discussion}

The regulation of the $\mathrm{K}_{\mathrm{ATP}}$ channel is complex. In addition to known small molecule modulators like ATP, ADP, sulfonylureas, etc., $\mathrm{K}_{\mathrm{ATP}}$ channel function is regulated by expression, trafficking, and turnover in the plasma membrane. A key-regulatory element within the channel protein is the ER retention signal, RKR, in the C-terminal domain of both Kir6.2 and SUR $1^{37}$. RKR serves as a point of control to ensure that only appropriately assembled channels with the correct stoichiometry and subunit composition traffic to the plasma membrane $\mathrm{r}^{37}$.

Though truncated Kir6.2 mutants are known to form functional, ligand gated $\mathrm{K}^{+}$channels, the effects of truncation on the orientation of Kir6.2 channels in the plasma membrane in the absence of SURx have not been explored. To further investigate the role of the RKR sequence in the regulation of $\mathrm{K}_{\mathrm{ATP}}$ channels, the orientation of Kir6.2 mutants and the resulting functional implications, we utilized a combination of immunocytochemistry and electrophysiology of mammalian cells and Xenopus oocytes transfected with Kir6.2 mutants. For these studies, three constructs were prepared using wild-type Kir6.2 as template. Figure 1 shows a schematic representation of the (i) wild type Kir6.2; (ii) a C-terminal truncated Kir6.2, (iii) Kir6.2 $\Delta$ C26 with an N-terminal hexahistidine (6xHis) tag (6xHis-Kir6.2 $\Delta$ C26), and (iv) N-terminal EGFP-chimera of Kir6.2 $\Delta$ C26 (6xHis-EGFP-Kir6.2 $\Delta$ C26). These constructs were previously shown to generate an ATP-sensitive $\mathrm{K}^{+}$current in oocytes and HEK cells ${ }^{26,44,47}$. Furthermore, the 6xHis tag allows for utilization of commercial antibodies raised against the tag to detect Kir6.2 mutants as well as facilitating potential purification of the protein in future applications ${ }^{47}$. Constructs were expressed in oocytes or HEK293, cells that lack native $\mathrm{K}_{\text {ATP }}$ channel background expression.

In Xenopus oocytes, addition of high concentrations of ATP revealed a population of $\mathrm{K}^{+}$channels with a single channel amplitude of $-2.3 \pm 0.5 \mathrm{pA}$ (Fig. $2 \mathrm{~A}, n=3$ ), in addition to the population with an expected amplitude of $-3.8 \pm 0.3 \mathrm{pA}(n=3)$. Due to the inward rectification properties of Kir6.2, it is predicted that inverted channels with outward oriented $\mathrm{N}$ - and $\mathrm{C}$-termini have a single channel amplitude, corresponding to an inverted rectification, of around $-2 \mathrm{pA}^{26}$, in good agreement with the observed second population of channel openings (Fig. 2B). In addition, when exposing outside-out patches from oocytes expressing Kir6.2 $\Delta \mathrm{C} 26$ to high concentration of extracellular ATP, we observed an inhibitory effect on channel activity (Fig. 2C), that likely results from exposure of the intracellular face to the extracellular milieu. It was impossible to determine with certainty if all channels, both normally oriented and abnormally inserted, were closed at that zero current level. Hence, there is a risk for underestimating the effect of extracellular ATP in these trials. However, when Kir6.2DC26 was co-expressed with SUR1, no effect of extracellular ATP is seen (Fig. 2D).

To evaluate the expression and orientation of Kir6.2 subunits, immunocytochemistry was performed on transfected HEK293 cells and non-transfected controls. In wild-type Kir6.2, both the N- and C-termini of the protein are found on the cytoplasmic face of the cell membrane ${ }^{2}$. Thus, no expression of $\mathrm{N}$ - or $\mathrm{C}$ - terminal epitopes should be observed when antibodies towards these regions are introduced to the exterior of the cell. Extracellular localization of the N-terminal 6xHis tag or EGFP was investigated in non-permeabilized cells, whereas total Kir6.2 membrane expression was evaluated in permeabilized cells, which allows access to antigens present on both the extracellular and cytoplasmic side of the membrane. Previous immunohistochemistry experiments have relied on hemagglutinin (HA) tags inserted into the Kir6.2 protein in the extracellular loop of the protein, facilitating detection of surface expression ${ }^{37}$. Importantly, these protocols were not extended to explore the possibility of inverted channels, thus potentially inverted channels were not studied. The protocol employed herein facilitates assessment of both protein expression and orientation of the varying constructs as outlined below.

Figure 3 shows typical fluorescence images obtained using HEK293 cells transfected with $6 x H i s-K i r 6.2 \Delta C 26$. No intrinsic fluorescence at the wavelengths utilized was observed from the $6 x H i s-K i r 6.2 \Delta \mathrm{C} 26$ chimera, thus staining is required for visualization. For this task, biotinylated anti-6xHis was labeled with streptavidin-fluorescein conjugate. Expression of $6 x H i s-K i r 6.2 \Delta \mathrm{C} 26$ was observed in both intact and permeabilized cells, suggesting that accessible $6 \mathrm{xHis}$ moieties are present on both sides of the plasma membranes. In contrast, examination of wild-type, non-transfected HEK293 cells revealed no background membrane staining and thus low non-specific 
A

Kir6.2 2 C26 $1 \mathrm{mM} \mathrm{ATP}$

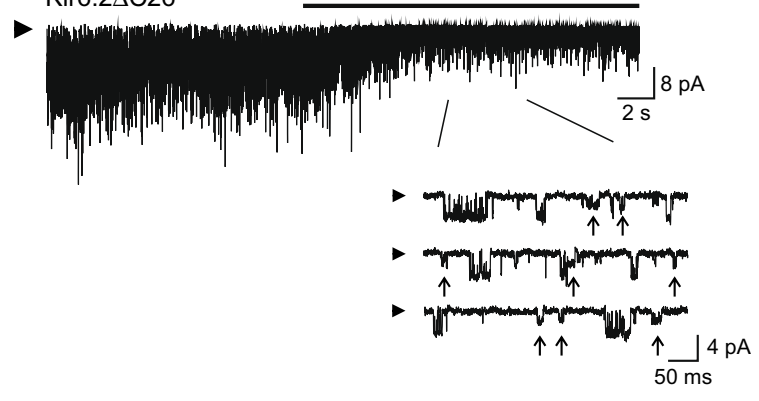

B

Kir6.2AC26

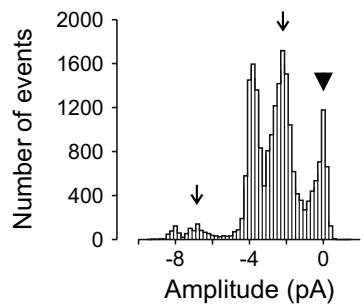

Kir6.2AC26 + SUR1

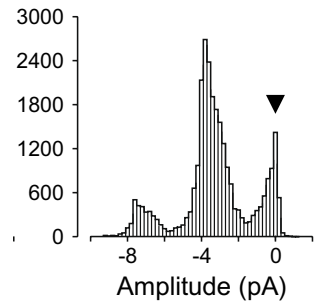

C

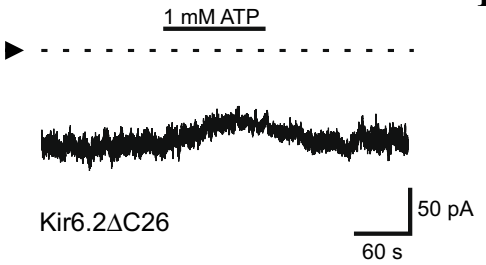

D

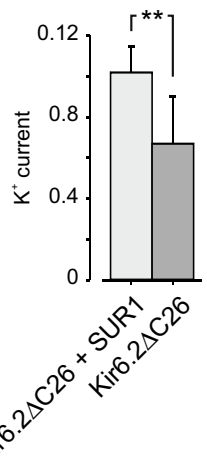

Figure 2. SUR1 affects orientation of Kir6.2 in the membrane. (A) Representative inside-out recording of channel currents from an oocyte injected with mRNA encoding Kir6.2 $\Delta$ C26. Inset shows channel activity at an expanded time scale. Two populations of channel openings are observed, $-3.8 \pm 0.5 \mathrm{pA}$ and $-2.2 \pm 0.3 \mathrm{pA}$ (arrow). (B) Amplitude histogram of inside-out current traces from patches excised from oocytes injected with Kir6.2 $\Delta$ C26 alone (left) or together with SUR1 (right). Data were obtained in the presence of $1 \mathrm{mM}$ ATP in the bath solution (i.e. intracellular side). Arrow indicates additional population of channel openings seen in the absence of SUR1. A total of $22 \times 10^{3}$ and $38 \times 10^{3}$ events were obtained, respectively. (C) Representative recordings from Kir6.2 $\Delta$ C26 channel activity from an outside-out patch, subsequently exposed to $1 \mathrm{mM}$ ATP. (D) Summary of outside-out patches from oocytes injected with Kir6.2 $\Delta$ C26 + SUR1 $(n=3)$ and Kir6.2 $\Delta 26$ $(n=4)$, and the effect of extracellularly $1 \mathrm{mM}$ ATP expressed as the ratio between the current measured before and during ATP. Arrowhead indicated zero current level, error bars are $\pm \mathrm{SD}$ and ${ }^{\star *}$ represents $P<0.01$.

adsorption of reagents, demonstrating the reliability of cytochemical detection of inverted channels (Supporting Information, Fig. S1). For all immunocytochemistry, at least 10 successful transfections were analyzed, with at least 10 images per successful transfection.

In contrast, expression of $6 \mathrm{xHis}-\mathrm{EGFP}-\mathrm{Kir} 6.2 \Delta \mathrm{C} 26$ exhibited a markedly different orientation profile. Figure 4 shows fluorescence images obtained using HEK293 cells transfected with $6 x$ His-EGFP-Kir6.2 $\Delta$ C26. As anticipated, EGFP fluorescence was observed in all cells regardless of permeabilization. The orientation of EGFP was investigated using anti-EGFP-AlexaFluor 594 conjugate since spectral overlap of EGFP and fluorescein limited the use of streptavidin-fluorescein conjugates and streptavidin with red-shifted labels exhibited high non-specific staining. Upon staining, clear differences in the distribution of EGFP orientation were observed. No significant staining of EGFP was observed in intact cells, whereas AlexaFluor 594 signal was observed in all permeabilized cells. Combined, these data suggest that the EGFP moiety, and thus the N-terminus of the Kir6.2 chimera, is expressed on the cytoplasmic side of the cell membrane as is found in wild-type $\mathrm{K}_{\mathrm{ATP}}$ channels, with little or no inversion of the channel proteins. 
Intact cells
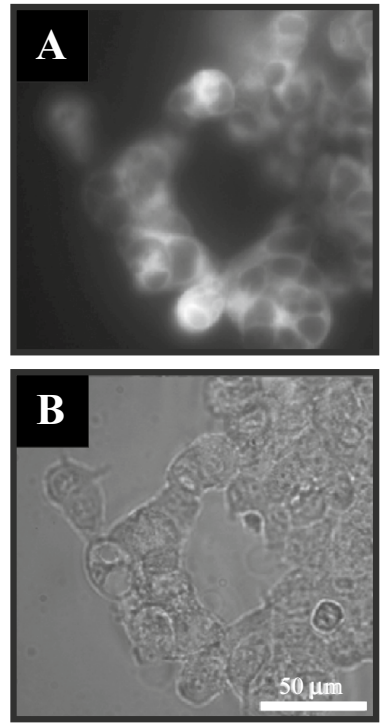

\section{Permeablized cells}
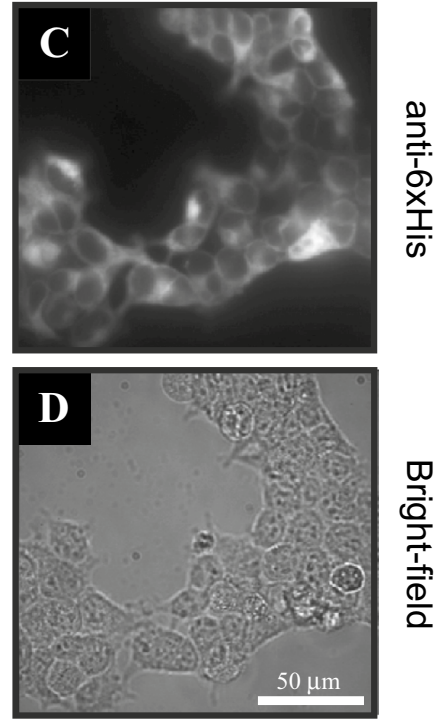

Figure 3. Immunocytochemistry of HEK293 cells transfected with $6 x H i s-K i r 6.2 \Delta C 26$. Intact $(\mathbf{A}, \mathbf{B})$ and permeabilized $(\mathbf{C}, \mathbf{D})$ cells were utilized to explore the orientation of $6 \mathrm{xHis}-\mathrm{Kir} 6.2 \Delta \mathrm{C} 26$ within the cell membrane. Biotinylated anti-6xHis was observed using streptavidin-fluorescein conjugate. Bright-field images (B and $\mathbf{D})$ are provided for reference, and all images are on the same intensity scale.

Intact cells
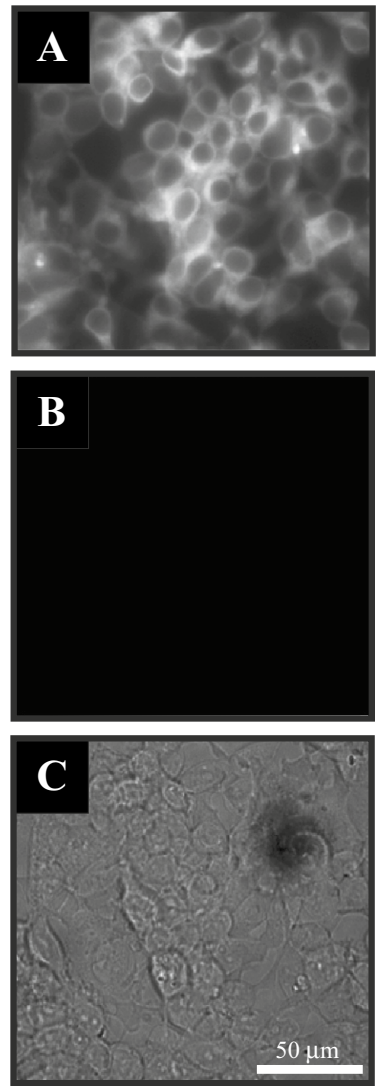

\section{Permeablized cells}

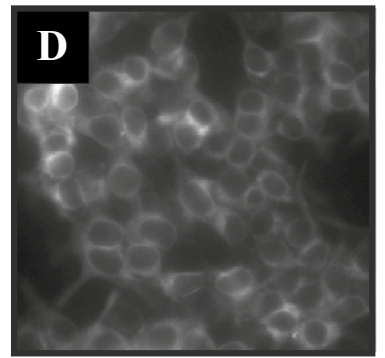

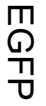
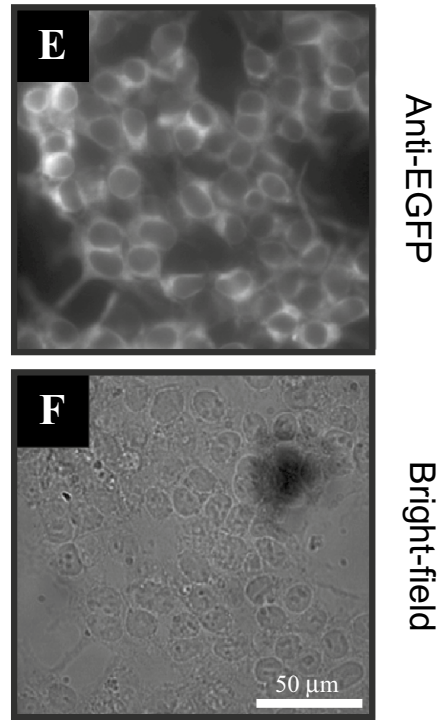

Figure 4. Immunocytochemistry of HEK293 cells transfected with 6xHis-EGFP-Kir6.2 $\Delta$ C26. Intact (A-C) and permeabilized (D-F) cells were utilized to explore the orientation of $6 x$ His-EGFP-Kir6.2 $\Delta$ C26 within the cell membrane. EGFP fluorescence (A and D) was observed under all conditions, whereas fluorescence resulting from anti-EGFP-AlexaFluor 594 conjugate was only observed in permeabilized cells (B and E). Bright-field images $(\mathbf{C}$ and $\mathbf{F})$ are provided for reference. All fluorescent images are on the same intensity scale. 
To correlate the function of Kir6.2 $\Delta \mathrm{C} 26$ subunits outlined above with membrane orientation, electrophysiological characterization of HEK293 cells transfected with either 6 xHis-Kir6.2 $\Delta$ C26 or $6 x$ His-EGFP-Kir6.2 $\Delta$ C26 was performed using whole-cell recordings (Fig. 5). Though whole-cell recordings monitor the net macroscopic current for the entire cell, the capability to monitor $\mathrm{K}^{+}$flux in the presence and absence of membrane side-specific Kir6.2 modulators facilitates investigation of orientation. Non-transfected and transfected HEK293 cells were chosen for these experiments as they do not natively express $\mathrm{K}_{\mathrm{ATP}}$ channels and typically have low total ion currents prior to transfection. Whole-cell currents were measured when the cells were immersed in extracellular solution, followed by exposure to $1 \mathrm{mM}$ ATP on the cytoplasmic side, a concentration sufficient to inhibit $>90 \%$ of $\mathrm{K}^{+}$flux via Kir6.2 $\Delta \mathrm{C} 26^{3,4,26,47}$. As seen in Fig. $5 \mathrm{~A}, \mathrm{~B}$, exposure to $1 \mathrm{mM}$ ATP reduced the net current by ca. $60 \%$ in cells transfected with $6 x$ His-Kir6.2 $\Delta$ C26. Importantly, the binding site for ATP resides on the cytoplasmic side of the wild-type $\mathrm{K}_{\mathrm{ATP}}$ channel, and ATP does not readily cross the cell membrane. When ATP was removed from the solution, whole-cell currents returned to their previous values. Thus, reversible inhibition by ATP is supportive of inverted channels within the membrane. In a similar experiment performed on cells transfected with $6 x$ His-EGFP-Kir6.2 $\Delta \mathrm{C} 26$, no statistically significant decrease in $\mathrm{K}^{+}$currents were observed. However, larger

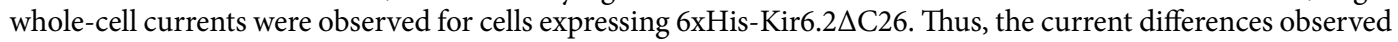
are likely due to increased protein expression and/or trafficking of $6 x$ His-Kir6.2 $\Delta \mathrm{C} 26$ compared to the larger $6 x$ His-EGFP-Kir6.2 $\Delta$ C26. Interestingly channel activity was retained even in the presence of physiological Ca ${ }^{2+}$ levels in the extracellular solution. Wild-type $\mathrm{K}_{\mathrm{ATP}}$ channels are phosphorylated by intracellular PKA and PKC, exposed to adequate cytoplasmic milieu such as low $\mathrm{Ca}^{2+}$, and associated with phosphatidylinositols, which may also lower currents.

To further explore this phenomenon, cells were exposed to thimerosal, a membrane impermeant oxidizing agent. Previous studies showed that exposure of the intracellular face of wild-type $K_{\text {ATP }}$ channels residing in excised membrane patches to thimerosal resulted in loss of channel activity that could be partially reversed upon exposure to a suitable reducing agent, e.g. DTT ${ }^{48}$. Exposure of cells transfected with $6 x \mathrm{His}-\mathrm{Kir} 6.2 \Delta \mathrm{C} 26$ to thimerosal resulted in a ca. $60 \%$ reduction in current, approximately $50 \%$ of which was restored upon exposure to DTT, in good agreement with previous work ${ }^{48}$, at which point whole-cell currents were unchanged upon exposure to buffer (Fig. 5). Conversely, no loss of channel activity was observed in cells transfected with 6xHisEGFP-Kir6.2 $\Delta \mathrm{C} 26$ upon exposure to thimerosal nor was activity enhanced upon exposure to DTT. When these same cells were exposed again to ATP, a ca. $60 \%$ decrease in activity was observed for cells expressing 6 xHisKir6.2 $\Delta$ C26. Exposure to inhibiting concentrations of tolbutamide yielded no statistically significant differences in whole-cell currents for either construct, an expected result since SUR1 is not expressed in these cells. For non-transfected, wild-type HEK293 control cells, sustained low level currents, likely from endogenous ion channel expression, were observed in all solutions tested with no statistically significant changes upon application of $\mathrm{K}_{\text {ATP }}$ channel modulators (Fig. 5B), supporting the low $\mathrm{K}_{\text {ATP }}$ background conductance in these cells. HEK293 cells endogenously express $\mathrm{P} 2 \mathrm{Y}$ receptors ${ }^{49}$, which could be potentially activated to decrease $\mathrm{K}_{\text {ATP }}$ channel activity by reducing $\mathrm{PIP}_{2}$ near the $\mathrm{K}_{\mathrm{ATP}}$ channel ${ }^{39,50}$. However, since no effect of extracellular applied ATP on $\mathrm{K}^{+}$

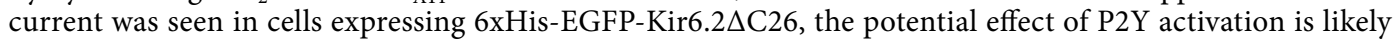
small. Finally, the current measured for $6 \mathrm{xHis}-\mathrm{Kir} 6.2 \Delta \mathrm{C} 26$ under these conditions is approximately $2 \times$ that for $6 \mathrm{xHis}$-EGFP-Kir6.2 $\Delta \mathrm{C} 26$. Since the mean channel conductance is similar for both constructs, the observed difference in current likely results from higher expression and/or membrane trafficking of the significantly smaller 6xHis-Kir6.2 $\Delta \mathrm{C} 26$.

Though immunocytochemical staining alone might reveal non-functional monomers or oligomers of Kir6.2 that are inadvertently transported to the plasma membrane, the ligand-modulated $\mathrm{K}^{+}$currents that are observed upon extracellular addition of ligands strongly support the functional expression of an inverted $\mathrm{K}^{+}$channel within the cell membrane. Combined, the electrophysiological and immunocytochemical data support the hypothesis that Kir6.2 $\Delta$ C26 transfected into mammalian cells results in a sub-population of inverted ion channels in the plasma membrane that retain ligand sensitivity and ion conductance.

In wild-type $\mathrm{K}_{\mathrm{ATP}}$ channels, the RKR retention signal in both Kir6.2 and SUR1 at the cytoplasmic face provides a quality control check that ensures the channels are properly assembled in the ER before further trafficking to the Golgi apparatus ${ }^{36,37}$. Truncated Kir6.2 mutants that lack the RKR sequence are capable of trafficking to the membrane allowing this quality control checkpoint to be bypassed. Our data support the hypothesis that not only are Kir6.2 homologous channels able to exit the ER and traffic through the Golgi apparatus to the plasma membrane, but that they may form functional channels that are inserted into the plasma membrane in an inverted orientation. Interestingly, the attachment of a large, water-soluble protein domain, EGFP in this case, located at the N-terminus significantly modulates the orientation of the protein, resulting in normal orientation and regulation of the channel within the plasma membrane. We hypothesize that these differences in orientation result from changes in post-translation processing of the proteins within the ER. EGFP serves to significantly change the orientation of the protein, most likely by introducing a large energetic barrier for protein orientation, resulting in unidirectional insertion (Fig. 6).

Lastly, there are many potential binding sites for $\mathrm{PIP}_{2}$ on Kir6.2, several of which are located in the C-terminus ${ }^{39}$. The mechanism for how $\mathrm{PIP}_{2}$ interacts and affects channel activity is not entirely clear, but a possible effect with the C-terminal truncation of Kir6.2 is that the channel is activated partly because of its altered PIP interaction. In that case, it could also explain why truncated Kir6.2 can be open even if abnormally inserted into the plasma membrane. A reasonable control would have been full-length Kir6.2. However, this construction does not generate $\mathrm{K}^{+}$conductance without co-expression of SUR1, and hence, co-expression with SUR1 results in truncated Kir6.2 being normally inserted. Shorter truncations of Kir6.2, like C14 and C18, resulted in low $\mathrm{K}^{+}$conductance ${ }^{26}$, so it is likely that the RKR (AA369-371) included in the deletion C26, and no other positively charged amino acids in the c-terminal region (Arg-377, Arg-379, and Arg-381) are the main explanation for abnormal insertion. The impact of the His-tag fused to Kir6.2 $\Delta$ C26 (construct iii, Fig. 1 ) is difficult to 
A
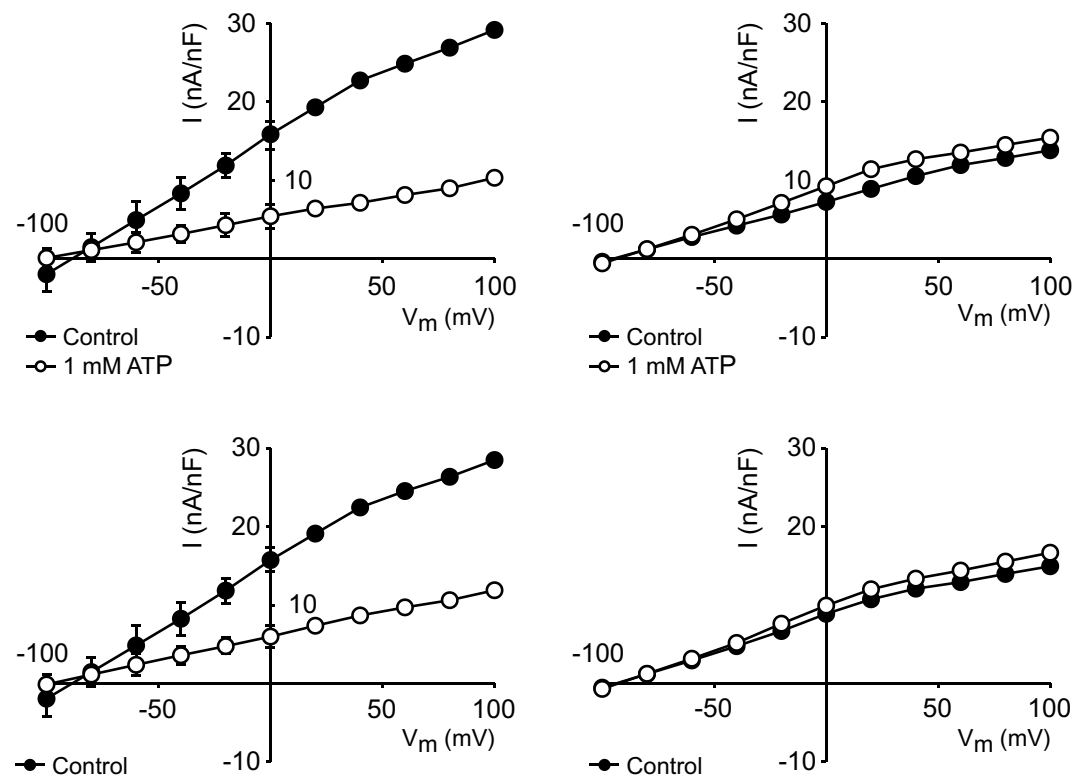

$-0-10 \mu \mathrm{M}$ thimerosal

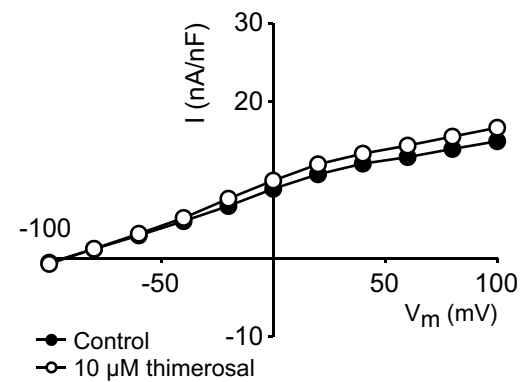

B

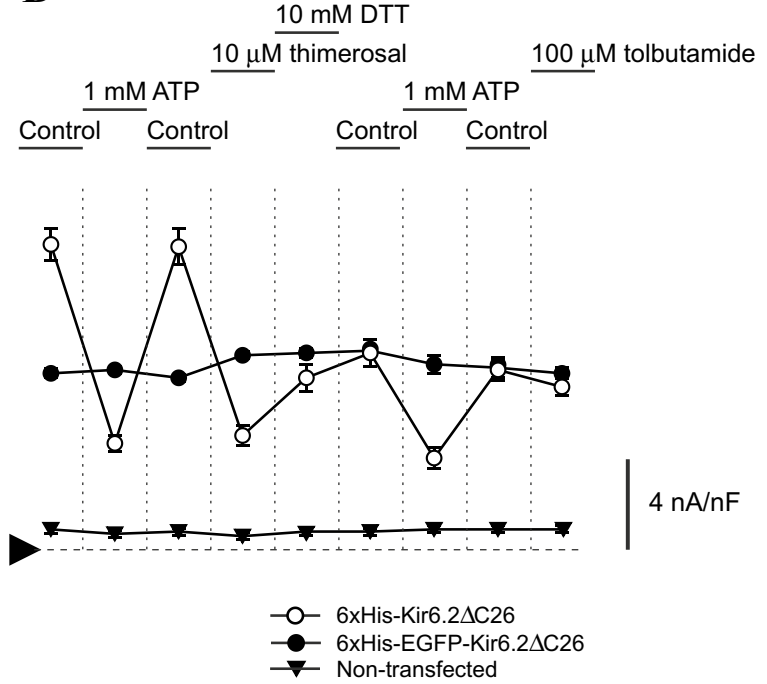

C

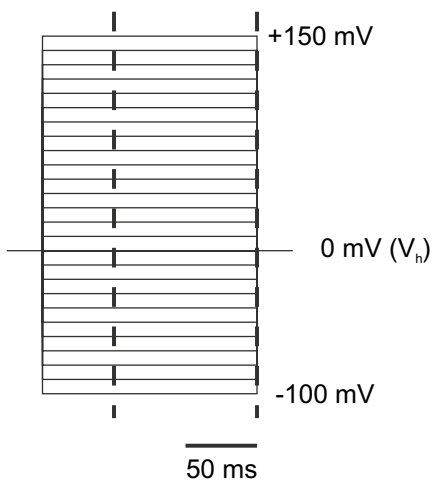

Figure 5. Whole-cell currents recorded from HEK293-cells expressing of different Kir6.2 variants. (A) Current-voltage (I-V) relationships of whole-cell currents in the presence of extracellular solution (denoted control), and $1 \mathrm{mM}$ ATP or $10 \mu \mathrm{M}$ thimerosal, as indicated. The cells were voltage-clamped at $0 \mathrm{mV}$ (holding potential $\mathrm{V}_{\mathrm{h}}$ ) for $25 \mathrm{~ms}$ prior to and following each pulse, and subsequently pulsed in steps of $+10 \mathrm{mV}$ for $150 \mathrm{~ms}$, starting from $-100 \mathrm{mV}$ to $+150 \mathrm{mV}$. (B), summary of normalized whole-cell currents of $6 \mathrm{xHis}-$ Kir6.2 $\Delta$ C26 (open circles), 6xHis-EGFP-Kir6.2 $\Delta$ C26 (filled circles), and non-transfected HEK293 (filled triangles) recorded at $0 \mathrm{mV}$. Whole-cell currents were normalized using membrane capacitance to compensate for variations in cell size. Each recording represents consecutive exposures to control, $1 \mathrm{mM}$ ATP, control, $10 \mu \mathrm{M}$ thimerosal, $1 \mathrm{mM}$ DTT, control, $1 \mathrm{mM}$ ATP, control, and finally $100 \mu \mathrm{M}$ tolbutamide. Arrowhead indicates zero current level, and error bars are \pm SD. $n=10$ for each cell type. (C) Mean currents were measured from 50 to $150 \mathrm{~ms}$ (dashed lines) and were plotted versus applied potential. Background current measured in transfected HEK293 cells was subtracted from mean currents, and plotted under the assumption that $E_{K}$ is $-83 \mathrm{mV}$ in the solutions used. Only $-100 \mathrm{mV}$ to $+100 \mathrm{mV}$ is presented, in $+20 \mathrm{mV}$ incremental steps, in the I-V relationships in $(\mathbf{A})$. 


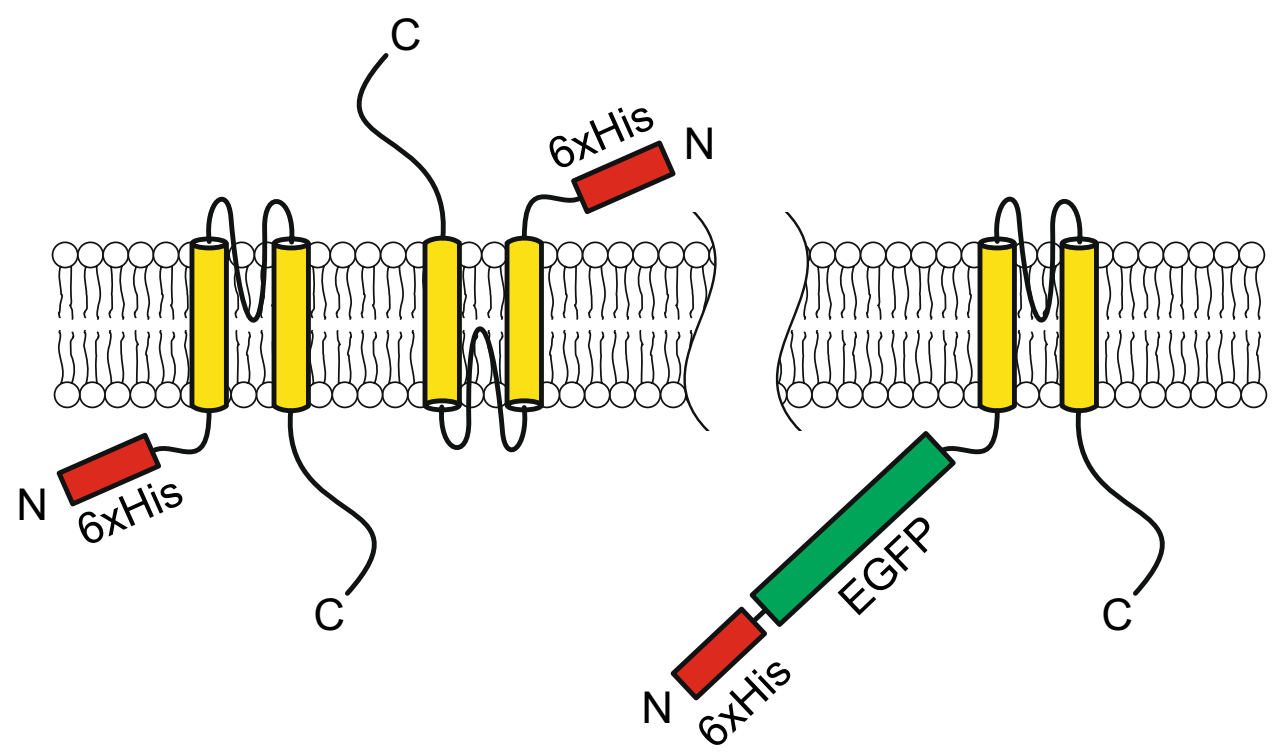

Figure 6. Schematic representation of protein orientation. Immunocytochemical and electrophysiological data support a model where Kir6.2 $\Delta \mathrm{C} 26$, which lacks native ER retention and membrane trafficking signals, is abnormally inserted in the plasma membrane (left), suggesting abnormal trafficking. Conversely, inclusion of a large, water-soluble domain on the $\mathrm{N}$-terminus imparts a sufficient energy barrier such that all the proteins are inserted in a unidirectional manner, overcoming the lack of ER retention and trafficking signals (right).

assess, but it appears not to be sufficient to orient the Kir in the normal position as the inclusion of EGFP does (construct iv, Fig. 1).

The potential biological significance of these observations is currently unknown. However, mutations in $\mathrm{K}_{\text {ATP }}$ channels that alter trafficking to the plasma membrane have been linked to $\mathrm{PHHI}^{32}$. It is possible that as yet unknown trafficking, assembly and orientation mechanisms may play a role in $\mathrm{K}_{\mathrm{ATP}}$ channel defects associated with abnormal physiological function. The fact that a protein can be translated, trafficked and expressed in a cell membrane within a mammalian cell line in an inverted fashion presents a number of intriguing possibilities for altered biological function that should be further investigated.

\section{Conclusion}

The data presented in this research report is the first observations of inverted ATP-sensitive $\mathrm{K}^{+}$channels within cellular expression models. Truncated mutants of Kir6.2, when expressed alone, were found to yield randomly inserted channel complexes, both normal and abnormal insertions, that were modulated in a cell surface specific manner upon application of ligands to the extracellular side of the channel. Abnormal channel population was not seen when co-expressed with SUR1. Fusion expression of a large, water-soluble protein domain like EGFP, to the cytoplasmic N-terminal domain of the channel resulted in channels that were correctly oriented within the cell membrane. We postulate that the lack of the RKR ER retention signal in the truncated Kir6.2 mutants used in our experiments prevents proper quality control and that the RKR sequence plays a key role in channel orientation in addition to channel assembly during the translation and trafficking processes. While the biological significance remains unknown, the existence of abnormally inserted channels presents a number of interesting possibilities for defective biological function and warrants further investigation. Further, these data suggest the need for caution in interpreting results from cellular studies relying on truncated Kir6.2 mutants and other truncated sequences, as well as potential effects on protein orientation of membrane proteins tagged with fluorescent proteins. Finally, expression of inverted channels may present unique opportunities to investigate compounds that may modulate Kir6.2 but are membrane impermeant, thus avoiding the need for injection or other delivery platforms to study the effects of such compounds.

Received: 18 May 2021; Accepted: 6 October 2021

Published online: 02 November 2021

\section{References}

1. Seino, S. ATP-sensitive potassium channels: A model of heteromultimeric potassium channel/receptor assemblies. Annu. Rev. Physiol. 61, 337-362 (1999).

2. Clement, J. P. 4th. et al. Association and stoichiometry of $\mathrm{K}_{\mathrm{ATP}}$ channel subunits. Neuron 18, 627-638 (1997).

3. Aguilar-Bryan, L. \& Bryan, J. Molecular biology of adenosine triphosphate-sensitive potassium channels. Endocr. Rev. 20, 101-135 (1999).

4. Aguilar-Bryan, L. et al. Toward understanding the assembly and structure of KATP channels. Physiol. Rev. 78, 227-245 (1998). 
5. Ashcroft, F. M., Harrison, D. E. \& Ashcroft, S. J. H. Glucose induces closure of single potassium channels in isolated rat pancreatic beta-cells. Nature 312, 446-448 (1984).

6. Ashcroft, F. M. \& Rorsman, P. ATP-sensitive K+ channels: A link between B-cell metabolism and insulin secretion. Biochem. Soc. Trans 18, 109-111 (1990).

7. Inagaki, N. et al. Reconstitution of I-KATP: An inward rectifier subunit plus the sulfonylurea receptor. Science 270, 1166-1170 (1995).

8. Ashcroft, F. M. \& Rorsman, P. Molecular defects in insulin secretion in type-2 diabetes. Rev. Endocr. Metab. Disord. 5, 135-142 (2004).

9. Taniguchi, J., Noma, A. \& Irisawa, H. Modification of the cardiac action-potential by intracellular injection of adenosine-triphosphate and related substances in guinea-pig single ventricular cells. Circ. Res. 53, 131-139 (1983).

10. Babenko, A. P., Gonzalez, G., Aguilar-Bryan, L. \& Bryan, J. Reconstituted human cardiac K-ATP channels: Functional identity with the native channels from the sarcolemma of human ventricular cells. Circ. Res. 83, 1132-1143 (1998).

11. Noma, A. ATP-regulated $\mathrm{K}^{+}$channels in cardiac muscle. Nature 305, 147-148 (1983).

12. Spruce, A. E., Standen, N. B. \& Stanfield, P. R. Voltage-dependent ATP-sensitive potassium channels of skeletal-muscle membrane. Nature 316, 736-738 (1985).

13. Standen, N. B. et al. Hyperpolarizing vasodilators activate ATP-sensitive K+ channels in arterial smooth-muscle. Science 245, $177-180$ (1989).

14. Ashford, M. L. J., Sturgess, N. C., Trout, N. J., Gardner, N. J. \& Hales, C. N. Adenosine-5' triphosphate-sensitive ion channels in neonatal rat cultured central neurons. Pflugers Arch. 412, 297-304 (1988).

15. Cook, D. L. \& Hales, C. N. Intracellular ATP directly blocks K+ channels in pancreatic b-cells. Nature 311, 271-273 (1984).

16. Nichols, C. G., Ripoll, C. \& Lederer, W. J. ATP-sensitive potassium channel modulation of the guinea pig ventricular action potential and contraction. Circ. Res. 68, 280-287 (1991).

17. Gasser, R. N. \& Vaughan-Jones, R. D. Mechanism of potassium efflux and action potential shortening during ischaemia in isolated mammalian cardiac muscle. J. Physiol. 431, 713-741 (1990).

18. Light, P. E. Cardiac K-ATP channels and ischemic preconditioning: Current perspectives. Can. J. Cardiol. 15, 1123-1130 (1999).

19. Gross, G. J. \& Peart, J. N. K-ATP channels and myocardial preconditioning: an update. Am. J. Physiol. Heart Circ. Physiol. 285, H921-H930 (2003).

20. Edwards, G. \& Weston, A. H. The pharmacology of atp-sensitive potassium channels. Annu. Rev. Pharmacol. Toxicol. 33, 597-637 (1993).

21. Clement, J. P. 4th. et al. Association and stoichiometry of K(ATP) channel subunits. Neuron 18, 827-838 (1997).

22. Shyng, S. L. \& Nichols, C. G. Octameric stoichiometry of the KATP channel complex. J. Gen. Physiol. 110(6), 655-664 (1997).

23. Moreau, C., Prost, A. L., Derand, R. \& Vivaudou, M. SUR, ABC proteins targeted by K-ATP channel openers. J. Mol. Cell. Cardiol. 38, 951-963 (2005).

24. Hilgemann, D. W. \& Ball, R. Regulation of cardiac Na+, Ca2+ exchange and K-ATP potassium channels by PIP2. Science 273, 956-959 (1996).

25. Baukrowitz, T. \& Fakler, B. KATP channels gated by intracellular nucleotides and phospholipids. Eur. J. Biochem. 267, 5842-5848 (2000).

26. Tucker, S. J., Gribble, F. M., Zhao, C., Trapp, S. \& Ashcroft, F. M. Truncation of Kir6.2 produces ATP-sensitive K+ channels in the absence of the sulphonylurea receptor. Nature 387, 179-183 (1997).

27. Tanabe, K. et al. Direct photoaffinity labeling of Kir6.2 by [gamma-(32)P]ATP-[gamma]4-azidoanilide. Biochem. Biophys. Res. Commun. 272, 316-319 (2000)

28. Larsson, O., Deeney, J. T., Branstrom, R., Berggren, P. O. \& Corkey, B. E. Activation of the ATP-sensitive K+ channel by long chain acyl-CoA: A role in modulation of pancreatic beta-cell glucose sensitivity. J. Biol. Chem. 271, 10623-10626 (1996).

29. Li, N. et al. Structure of a pancreatic ATP-sensitive potassium channel. Cell 168(1-2), 101-110 (2017).

30. Ashcroft, F. M. \& Rorsman, P. ATP-sensitive K+ channels: A link between B-cell metabolism and insulin-secretion. Biochem. Soc. Trans. 18, 109-111 (1990).

31. Seino, S. \& Miki, T. Physiological and pathophysiological roles of ATP-sensitive K+ channels. Prog. Biophys. Mol. Biol. 81, 133-176 (2003).

32. Huopio, H., Shyng, S. L., Otonkoski, T. \& Nichols, C. G. K-ATP channels and insulin secretion disorders. Am. J. Physiol. Endocrinol. Metab. 283, E207-E216 (2002).

33. Ashcroft, F. M. Ion Channels and Disease (Academic Press, 2000).

34. Mannhold, R. K-ATP channel openers: Structure-activity relationships and therapeutic potential. Med. Res. Rev. 24, 213-266 (2004).

35. Taschenberger, G. et al. Identification of a familial hyperinsulinism-causing mutation in the sulfonylurea receptor 1 that prevents normal trafficking and function of K-ATP channels. J. Biol. Chem. 277, 17139-17146 (2002).

36. Sharma, N. et al. The c terminus of SUR1 is required for trafficking of KATP channels. J. Biol. Chem. 274, 20628-20632 (1999).

37. Zerangue, N., Schwappach, B., Jan, Y. N. \& Jan, L. Y. A new ER trafficking signal regulates the subunit stoichiometry of plasma membrane K-ATP channels. Neuron 22, 537-548 (1999).

38. Alekseev, A. E., Kennedy, M. E., Navarro, B. \& Terzic, A. Burst kinetics of co-expressed Kir6.2/SUR1 clones: Comparison of recombinant with native ATP-sensitive K+ channel behavior. J. Membr. Biol. 159(2), 161-168 (1997).

39. Haider, S., Tarasov, A. I., Craig, T. J., Sansom, M. S. \& Ashcroft, F. M. Identification of the PIP2-binding site on Kir6.2 by molecular modelling and functional analysis. $E M B O ~ J . ~ 26(16), 3749-3759$ (2007).

40. Larsson, O., Ammala, C., Bokvist, K., Fredholm, B. \& Rorsman, P. Stimulation of the KATP channel by ADP and diazoxide requires nucleotide hydrolysis in mouse pancreatic beta-cells. J. Physiol.Lond. 463, 349-365 (1993).

41. Gillis, K. D. et al. Effects of sulfonamides on a metabolite-regulated atpi-sensitive K+ channel in rat pancreatic b-cells. Am. J. Physiol. 257, C1119-C1127 (1989).

42. Bokvist, K., Rorsman, P. \& Smith, P. A. Block of ATP-regulated and Ca2+-activated K+ channels in mouse pancreatic beta-cells by external tetraethylammonium and quinine. J. Physiol. 423, 327-342 (1990).

43. Zilberter, Y., Burnashev, N., Papin, A., Portnov, V. \& Khodorov, B. Gating kinetics of ATP-sensitive single potassium channels in myocardial cells depends on electromotive force. Pflügers Arch. 411, 584-589 (1988).

44. Bränström, R. et al. Long chain coenzyme A esters activate the pore-forming subunit (Kir6.2) of the ATP-regulated potassium channel. J. Biol. Chem. 273, 31395-31400 (1998).

45. Rauf, F., Huang, Y., Muhandiramlage, T. P. \& Aspinwall, C. A. Analysis of protein kinase A activity in insulin-secreting cells using a cell-penetrating protein substrate and capillary electrophoresis. Anal. Bioanal. Chem. 397, 3359-3367 (2010).

46. Abramoff, M. D., Magalhaes, P. J. \& Ram, S. J. Image processing with ImageJ. Biophoton. Int. 11(7), 36-43 (2004)

47. Agasid, M. T. et al. Expression, purification, and electrophysiological characterization of a recombinant, fluorescent Kir6.2 in mammalian cells. Protein Expr. Purif. 146, 61-68 (2018).

48. Islam, M. S., Berggren, P. O. \& Larsson, O. Sulfhydryl oxidation induces rapid and reversible closure of the ATP-regulated K+ channel in the pancreatic beta-cell. FEBS Lett. 319, 128-132 (1993).

49. Schachter, J. B., Sromek, S. M., Nicholas, R. A. \& Harden, T. K. HEK293 human embryonic kidney cells endogenously express the P2Y1 and P2Y2 receptors. Neuropharmacology 36(9), 1181-1187 (1997). 
50. Kuo, A. et al. Crystal structure of the potassium channel KirBac1.1 in the closed state. Science 300, 1922-1926 (2003).

\title{
Acknowledgements
}

This work was supported by National Institute of Biomedical Imaging and Bioengineering of the National Institutes of Health under Award Nos. EB007047 and EB022297 and the National Science Foundation (0548167, 2003297). Financial support was also provided through the regional agreement on medical training and clinical research (ALF) between the Stockholm County Council and Karolinska Institutet; Swedish Society of Medicine (Bengt Ihre grant); the Swedish Research Council; the Novo Nordisk Foundation; the Swedish Diabetes Association; the Family Erling-Persson Foundation; the Skandia Insurance Company Ltd; Strategic Research Program in Diabetes at Karolinska Institutet; the Berth von Kantzow's Foundation; the Knut and Alice Wallenberg Foundation; Funds of Karolinska Institutet; Diabetes and Wellness Foundation; the Stichting af Jochnick Foundation; ERC-2018-AdG 834860 EYELETS.

\section{Author contributions}

B.H., R.B., O.L., I.L., B.L., S.N.Y., S.S., P.O.B., C.A. devised the project, the main conceptual ideas and proof outline. B.H., Y.H., W.Y., T.M., L.Q.C., J.Y. worked out the technical details, and performed the experiments and made the figures. All authors contributed in writing the manuscript.

\section{Funding}

Open access funding provided by Karolinska Institute.

\section{Competing interests}

The authors declare no competing interests.

\section{Additional information}

Supplementary Information The online version contains supplementary material available at https://doi.org/ 10.1038/s41598-021-00988-y.

Correspondence and requests for materials should be addressed to R.B.

Reprints and permissions information is available at www.nature.com/reprints.

Publisher's note Springer Nature remains neutral with regard to jurisdictional claims in published maps and institutional affiliations.

\begin{abstract}
(c) (i) Open Access This article is licensed under a Creative Commons Attribution 4.0 International cc) License, which permits use, sharing, adaptation, distribution and reproduction in any medium or format, as long as you give appropriate credit to the original author(s) and the source, provide a link to the Creative Commons licence, and indicate if changes were made. The images or other third party material in this article are included in the article's Creative Commons licence, unless indicated otherwise in a credit line to the material. If material is not included in the article's Creative Commons licence and your intended use is not permitted by statutory regulation or exceeds the permitted use, you will need to obtain permission directly from the copyright holder. To view a copy of this licence, visit http://creativecommons.org/licenses/by/4.0/.
\end{abstract}

(C) The Author(s) 2021 